\title{
Multicentric Myelolipoma in a Dog
}

\author{
Junichi KAMIIE ${ }^{1)}$, Keisuke FUEKI ${ }^{2)}$, Harumi AMAGAI ${ }^{2)}$, Youichiro ICHIKAWA ${ }^{2)}$ and Kinji SHIROTA ${ }^{1,3) *}$ \\ ${ }^{1)}$ Laboratory of Veterinary Pathology, School of Veterinary Medicine, Azabu University, 1-17-71 Fuchinobe, Sagamihara, Kanagawa \\ 229-8501, 2)Ichikawa Animal Hospital, 168-1 Nekiuti, Matsudo, Chiba 270-0011 and ${ }^{3)}$ Research Institute of Biosciences, Azabu \\ University, 1-17-71 Fuchinobe, Sagamihara, Kanagawa 229-8501, Japan
}

(Received 5 June 2008/Accepted 5 November 2008)

ABSTRACT. We report herein a case of multicentric myelolipoma in an 11-year-old beagle dog that presented with vomiting. Laparotomy demonstrated the presence of a large mass adherent to the greater omentum and multiple small white maculae in the spleen. Cytological and histological examinations revealed that the mass and maculae comprised mature adipocytes and hematopoietic elements including granulocytic, erythrocytic and megakaryocytic series in several phases of maturation and macrophages containing hemosiderin deposits, resembling bone marrow. Multicentric myelolipoma was diagnosed. This is first report of multicentric myelolipoma in a dog. KEY WORDS: canine, multicentric, myelolipoma.

Myelolipoma is a benign tumor comprising mature adipocytes associated with hematopoietic elements that resemble bone marrow. This tumor is rare in domestic animals, but common in the adrenal gland of elderly humans [3]. Few cases of myelolipoma have been reported in non-human primates [11], dogs [2, 7, 9, 12, 16-19], cats [6, 13] and birds [5]. In dogs, most cases of myelolipoma have been identified in the spleen $[2,12,16,19]$. Also, myelolipoma commonly arises as a single lesion [2, 7, 9, 12, 16-19]. Extrasplenic or multicentric myelolipomas in dogs are rare. We report herein a case of multicentric myelolipoma identified in the greater omentum and spleen of a dog.

An 11-year-old female beagle dog presented with vomiting and was admitted to a local veterinary clinic for physical examination. Serum biochemistry revealed high levels of alkaline phosphatase (ALP; >3500 U/L), glutamic pyruvic transaminase (GPT; $>1000 \mathrm{U} / \mathrm{L})$, glutamic oxaloacetic transaminase (GOT; $163 \mathrm{U} / \mathrm{L})$, total cholesterol $(>450 \mathrm{mg} / \mathrm{dL})$ and total bilirubin $(0.7 \mathrm{mg} / \mathrm{dL})$. Ultrasonography demonstrated a spherical and homogeneous mass $5.0 \mathrm{~cm}$ in diameter in the mid-abdomen. No other abnormalities were found in the abdominal organs or cavity. Adrenocorticotropic hormone (ACTH) stimulation test for Cushing's disease was performed and showed moderate elevation of serum cortisol $(22.1 \mathrm{mg} / \mathrm{dL})$. For pathological diagnosis of the abdominal mass, ultrasonography-guided aspirates were taken from the mass. Cytological examination of aspirates revealed the mass was formed of hematopoietic elements that included megakaryocytes, myelocytes, erythroblasts and a small number of lymphoblasts (Fig. 1). Based on these findings, the abdominal mass was diagnosed as myelolipoma.

Six weeks after cytological examination, levels of serum ALP and GOT were $1225 \mathrm{U} / \mathrm{L}$ and $95 \mathrm{U} / \mathrm{L}$, respectively, and laparotomy was performed under inhalational general anes-

\footnotetext{
* Correspondence to: Shirota, K., Laboratory of Veterinary Pathology, School of Veterinary Medicine, Azabu University, 117-71 Fuchinobe, Sagamihara, Kanagawa 229-8501, Japan. e-mail: shirota@azabu-u.ac.jp
}

thesia to extirpate the abdominal mass and clarify the nature of liver disorder. Laparotomy revealed a large, spheroid, soft, abdominal mass measuring $5.0 \times 4.5 \times 4.5 \mathrm{~cm}$. The mass was encapsulated in a membrane, well-circumscribed and adherent to the greater omentum. The cut surface of the mass revealed a solid appearance with dark red color, resembling bone marrow (Fig. 2). The lateral right lobe of the liver was enlarged, although no macroscopic abnormalities were apparent in other lobes. Laparotomy also revealed splenomegaly and some white maculae measuring up to 5.0 $\mathrm{mm}$ in diameter in the splenic head. These white maculae were well demarcated from surrounding tissue. The abdominal mass, spleen and lateral right lobe of the liver were excised for histopathological examination

Specimens were fixed in $10 \%$ phosphate-buffered formalin, processed routinely and embedded in paraffin. Paraffin section were cut at 3- $\mu \mathrm{m}$ thickness and stained with hematoxylin and eosin.

Histologically, the abdominal mass comprised mature adipocytes associated with hematopoietic elements that were composed of granulocytic, erythrocytic and megakaryocytic series in various phases of maturation (Figs. 3, 4). Macrophages containing hemosiderin were also identified within the hematopoietic tissue. These histological findings supported the diagnosis of myelolipoma on cytological examination.

White maculae of the spleen also consisted of adipocytes and hematopoietic elements, as seen in the abdominal mass (Fig. 5). Based on histological findings, splenic maculae were diagnosed as myelolipoma. Diffuse extramedullary hematopoiesis was observed outside of maculae in the spleen.

Histological examination of the lateral right lobe of the liver revealed swollen hepatocytes with clear cytoplasm, solitary necrosis of hepatocytes and infiltration of macrophages containing hemosiderin (Fig. 6). Accumulations of glycogen in hepatocytes were demonstrated by periodic acid-Schiff reaction. Mild lymphocytic infiltration was seen 


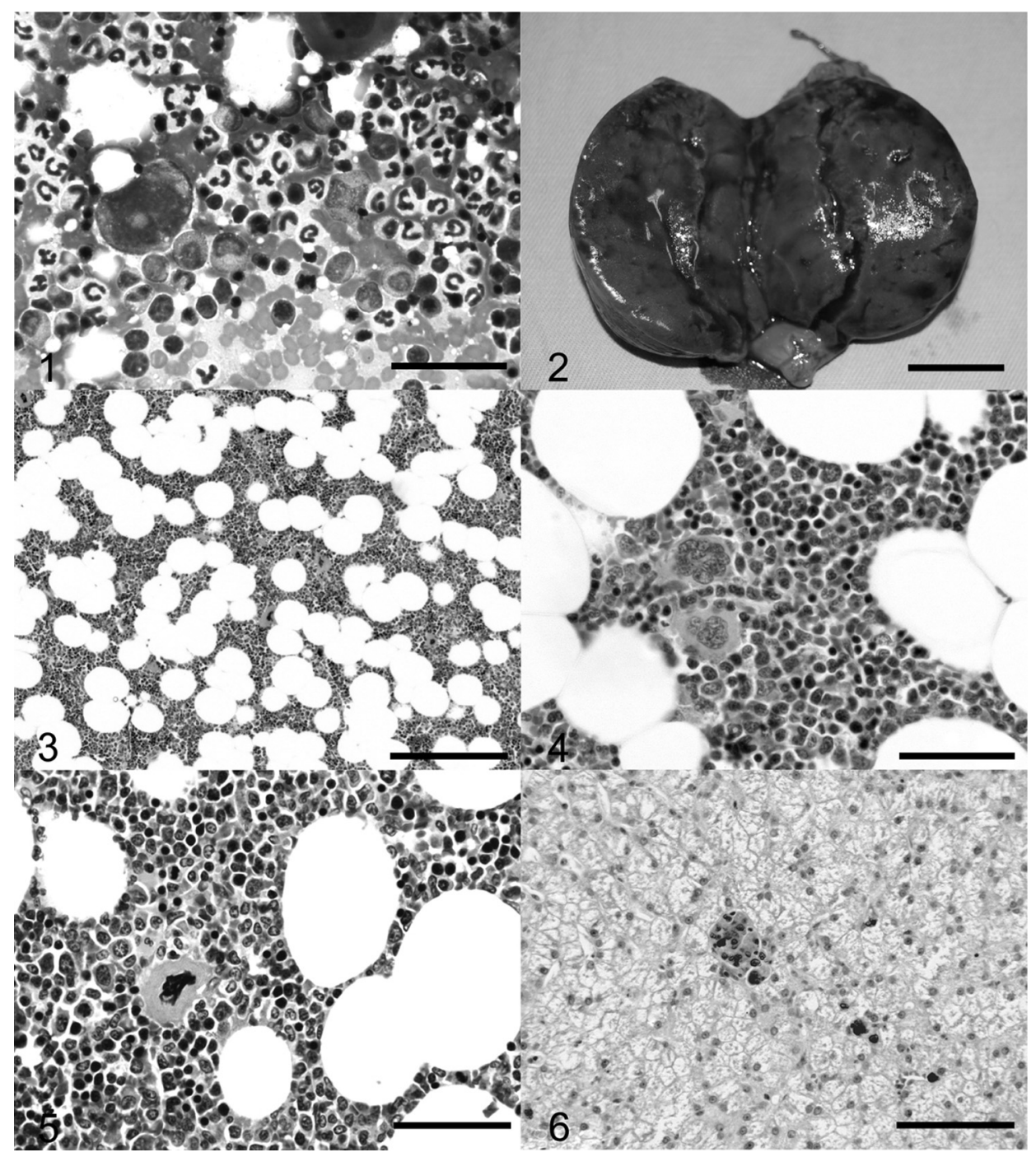

Fig. 1. Fine needle aspirate from the abdominal mass. Aspirate comprises adipocytes, megakaryocytes, myelocytes, erythroblasts and a small number of lymphoblasts. Diff quick stain. Bar, $50 \mu \mathrm{m}$.

Fig. 2. Cross-section of the abdominal mass. The cut surface of the mass shows a solid tumor with dark red color, like bone marrow. Bar, $3.0 \mathrm{~cm}$.

Fig. 3. The abdominal mass. The mass comprises mature adipocytes associated with various hematopoietic elements. Hematoxylin and eosin (HE) stain. Bar, $200 \mu \mathrm{m}$.

Fig. 4. The abdominal mass. Hematopoietic elements of the abdominal mass comprise granulocytic, erythrocytic and megakaryocytic series in several phases of maturation. HE stain. Bar, $50 \mu \mathrm{m}$.

Fig. 5. White maculae of the spleen, comprising mature adipocytes and several hematopoietic elements. HE stain. Bar, $50 \mu \mathrm{m}$.

Fig. 6. Lateral right lobe of the liver. Hepatocytes are swollen with clear cytoplasm. Infiltrations of macrophages containing hemosiderin are apparent. HE stain. Bar, $100 \mu \mathrm{m}$.

around the portal veins.

In the dog described here, tumors were found on the greater omentum and in the spleen independently, although most previously reported canine myelolipomas have occurred in the spleen $[2,12,16,19]$. The tissues in which myelolipoma frequently develops differ among species. Myelolipoma of the adrenal gland is most commonly found in humans [3] and non-human primates [11]. Myelolipoma in cats is usually found in the liver [6] or spleen [13]. The majority of myelolipomas in dogs occur in the spleen [2]. To date, 9 cases of myelolipomas in dogs have been reported $[2,7,9,12,16,17-19]$, with five of these cases occurring in the spleen $[2,12,16,19]$ and 2 cases in the adrenal gland [7, 17]. Myelolipoma in other organs is rare in the dog and has been reported in only 2 cases involving the spinal cord [9, 18]. In the present case, myelolipomas were found in the greater omentum and as multiple splenic lesions.

The term "multicentric tumor" denotes the tumor with 
multiple lesions, each of which is same tumor and has developed as a primary tumor. Myelolipoma is generally considered a benign tumor and metastasis to other organs has not been reported. The abdominal mass in the present case did not connect to the spleen and was well circumscribed with a distinct capsule. These findings suggest that tumors of the greater omentum and spleen in the present case developed independently. Therefore, we diagnosed this case as multicentric myelolipoma in a dog.

Canine myelolipomas in previous reports have been only a few millimeters in diameters, with the exception of a case involving a Cardigan Welsh Corgi [2]. Furthermore, no specific clinical signs that would suggest the presence of tumors have been reported in myelolipoma. These lesions are thus occasionally detected as an incidental finding during laparotomy or necropsy. A few cases of giant myelolipomas over 10 centimeters in diameter have been reported in humans [ 1 , 3 ], causing clinical symptoms including abdominal pain, nausea and vomiting and thus differing from the more typical asymptomatic cases of myelolipoma. In the case of the present dog, myelolipoma on the greater omentum formed a large mass over $4 \mathrm{~cm}$ in diameter. Vomiting in the present case might thus have been partially attributable to pressure on the gastrointestinal organs by the abdominal mass.

The present case showed high levels of serum ALP and GOT and pathological examination revealed hepatic glycogen degeneration. Hepatic glycogen degeneration in dogs is induced by either excess administration of steroids or hyperadrenocorticism due to adrenocortical hyperplasia or tumors. The dog in this case had not been administered steroids and displayed no gross macroscopic abnormalities of the adrenal gland. However, ACTH stimulation test showed a moderate increase in serum cortisol level and suggested the presence of Cushing's syndrome.

The pathogenesis of myelolipoma remains unclear. One hypothesis is based on embryonic hematopoiesis, which occurs diffusely throughout the peritoneal connective tissue and regresses with development of other hematopoietic tissues [4]. Recurrence of peritoneal hematopoiesis has been observed in several infectious conditions. This phenomenon could result from activation of dormant hematopoietic stem cells in the peritoneum that had been active in embryonic stage [4]. A similar mechanism could be ascribed to the myelolipoma of the greater omentum in this report. Prolonged stimulation with ACTH may also play a causative role in the development of myelolipoma [8, 15]. An increased frequency of myelolipoma has been shown in patients with excessive ACTH secretion, such as congenital adrenal hyperplasia [10]. In addition, injection of anterior pituitary extracts can induce myelolipomatous adrenal masses in rats [14]. In the present case, ACTH stimulation test showed a moderate increase in serum cortisol level, suggesting Cushing's syndrome. Present case showed multicentric occurrence of myelolipoma, although this tumor usually developes as a single lesion. Pathogenesis of multicentric occurrence was unknown in this dog, however, it might be possible that prolonged stimulation with ACTH had accumulated in the spleen and the remained embryonic hematopoietic tissue in the greater omentum, resulting in independent development of each myelolipoma in this case.

\section{REFERENCES}

1. Akamatsu, H., Koseki, M., Nakaba, H., Sunada, S., Ito, A., Teramoto, S. and Miyata, M. 2004. Giant adrenal myelolipoma: report of a case. Surg Today. 34: 283-285.

2. Al-Rukibat, R. K. and Bani Ismail, Z. A. 2006. Unusual presentation of splenic myelolipoma in a dog. Can. Vet. J. 47: 11121114.

3. Bovo, G., Picozzi, S. C., Vigano, P., Giuberti, A., Casu, M., Manganini, V., Mazza, L. and Strada, G. R. 2007. Giant adrenal myelolipoma: report of a case and review of the literature. Minerva Urol. Nefrol. 59: 455-458.

4. Fowler, M. R., Williams, R. B., Alba, J. M. and Byrd, C. R. 1982. Extra-adrenal myelolipomas compared with extramedullary hematopoietic tumors: a case of presacral myelolipoma. Am. J. Surg. Pathol. 6: 363-374.

5. Latimer, K. S. and Rakich, P. M. 1995. Subcutaneous and hepatic myelolipomas in four exotic birds. Vet. Pathol. 32: 84 87.

6. McCaw, D. L., da Silva Curiel, J. M. and Shaw, D. P. 1990. Hepatic myelolipomas in a cat. J. Am. Vet. Med. Assoc. 197: 243-244.

7. Morandi, F., Mays, J. L., Newman, S. J. and Adams, W. H. 2007. Imaging diagnosis-bilateral adrenal adenomas and myelolipomas in a dog. Vet. Radiol. Ultrasound. 48: 246-249.

8. Nagai, T., Imamura, M., Honma, M., Murakami, M. and Mori, M. 2001. 17alpha-hydroxylase deficiency accompanied by adrenal myelolipoma. Intern. Med. 40: 920-923.

9. Newman, S. J., Inzana, K. and Chickering, W. 2000. Extradural myelolipoma in a dog. J. Vet. Diagn. Invest. 12: 71-74.

10. Oliva, A., Duarte, B., Hammadeh, R., Ghosh, L. and Baker, R. J. 1988. Myelolipoma and endocrine dysfunction. Surgery 103: $711-715$

11. Porter BF., Goens SD., Brasky KM. and Hubbard GB. 2004. A case report of hepatocellular carcinoma and focal nodular hyperplasia with a myelolipoma in two chimpanzees and a review of spontaneous hepatobiliary tumors in non-human primates. J. Med. Primatol. 33: 38-47.

12. Prater, M. R., Bender, H. and Sponenberg, P. 1998. Intraabdominal mass aspirate from an aged dog. Vet. Clin. Pathol. 27: 54 .

13. Sander, C. H. and Langham, R. F. 1972. Myelolipoma of the spleen in a cat. J. Am. Vet. Med. Assoc. 160: 1101-1103.

14. Selye, H. and Stone, H. 1950. Hormonally induced transformation of adrenal into myeloid tissue. Am. J. Pathol. 26: 211-233.

15. Sieber, S. C., Gelfman, N. A., Dandurand, R. and Braza, F. 1989. Ectopic ACTH and adrenal myelolipoma. Conn. Med. 53: $7-10$.

16. Spangler, W. L., Culbertson, M. R. and Kass, P. H. 1994. Primary mesenchymal (nonangiomatous/nonlymphomatous) neoplasms occurring in the canine spleen: anatomic classification, immunohistochemistry, and mitotic activity correlated with patient survival. Vet. Pathol. 31: 37-47.

17. Tursi, M., Iussich, S., Prunotto, M. and Buracco, P. 2005. Adrenal myelolipoma in a dog. Vet. Pathol. 42: 232-235.

18. Ueno, H., Miyake, T., Kobayashi, Y., Yamada, K. and Uzuka, Y. 2007. Epidural spinal myelolipoma in a dog. J. Am. Anim. Hosp. Assoc. 43: 132-135.

19. Zimmer, M. A. and Stair, E. L. 1983. Splenic myelolipomas in two dogs. Vet. Pathol. 20: 637-638. 